B. THE LUNAR SURFACE AND ITS MORPHOLOGY 


\title{
THE GEOMORPHIC EVOLUTION OF \\ THE LUNAR SURFACE
}

\author{
L. B. RONCA \\ Lunar Science Institute, Houston, Texas, U.S.A.*
}

\begin{abstract}
Fundamental parameters of the geomorphology of a lunar surface are (i) the number and size distribution of craters, (ii) the degree of erosion of the craters and (iii) thickness and other characteristics of the regolith. These parameters are not independent of one another; as one changes through time, the other two will also change in a statistically predictable way.

In the continuous degradation sequence, the relationship between the number of craters per unit area and the degree of erosion of the craters is continuous and monotonic. This sequence occurs in areas subjected to intermediate to small impacts, and eroded mainly by the impacts themselves plus other small-scale erosional processes. In areas subjected to large impacts and mare flooding the discontinuous degradation sequence is predominant..

The relationship of the first two parameters, the number of craters and the degree of erosion, with the third parameter, the regolith, is not simple and is not yet understood. It appears, however, that the geomorphological stage is more important than the mare-versus-highland dichotomy of the lunar surface.

The solution of the function relating craters of the continuous degradation sequence with degree of erosion was defined as the geomorphic index of the area. Studies of the geomorphic index of stratigraphic surfaces show that areas covered by considerable ballistic sediments have a geomorphic index which is not a monotonic function of time. On the other hand, areas covered almost exclusively by mare flooding show an index which is a monotonic function of the age of the flooding. As each mare surface shows a considerable range in indices, it is concluded that maria are covered by surfaces formed through a considerable length of time. By using Apollo 11 and 12 radiometric ages it is suggested that the time of mare flooding lasted on the order of one billion $\left(10^{9}\right)$ years.

The geomorphic index of highland surfaces shows a remarkable degree of order; i.e., the farther an area is inland from the mare shores, the higher will be the index. No explanation is given to this phenomenon, but is suggested that lunar erosion is not just a localized phenomenon centered on the locus of an impact, but has lateral trends of regional dimensions. Electrostatic transportation as suggested by Gold is a possible mechanism.
\end{abstract}

\section{Introduction}

Fundamental parameters of the geomorphology of a lunar surface are (i) the number and size distribution of craters, (ii) the degree of erosion of the craters, and (iii) thickness and other characteristics of the regolith. At least in first approximation, it is likely that these parameters are not independent of one another; i.e. as the number of craters increases, the degree of erosion of preexisting craters also increases and progressive changes occur in the regolith.

Considering the statistical nature of the phenomenon, it would be worth-while to develop a unit of measure which includes all three of the above parameters. This unit could describe the geomorphology of any area, and, being the result of three independent measurements, could reduce the unavoidable statistical fluctuations. Previous work (Ronca and Green, 1970) has led to the development of a function which relates the number density of craters (excluding ghost craters) to the number of these craters

* Present address: Dept. of Geology, Wayne State University, Detroit, Michigan 48202. 
which are essentially uneroded. The value of this function at different areas was defined as the geomorphic index of that area. The relationship between the geomorphic index (that is, the first two parameters of lunar geomorphology) and the regolith (the third parameter) was presented in Ronca (1971a). This relationship appears to be not monotonic, and it is not yet understood. It appears, however, that the geomorphic index is more important, as far as the regolith is concerned, than the mare-versushighland dichotomy of the lunar surface.

What is the geological meaning of the geomorphic index? It is a description of the geomorphic age of an area. To use an example, a river system on the Earth is variously referred to as being in its youthful, mature, or old stage. This means that there is a sequence of characteristics which a river system displays consecutively from the time of its formation. The same concept can be applied to a lunar surface, from the time of its formation, when presumably no or only endogenous craters are present, to the time when the surface is completely covered with impact craters, many of which are highly eroded. The geomorphic index is the position of the lunar surface in this progression.

It is important to realize the basic difference between geomorphic age and geologic age. Just as on Earth one river may reach its old stage in a much shorter time than another river in a different area, so on the Moon one surface may reach a high geomorphic index sooner than another. Geologic age, on the other hand, is simply the age in years (or its position in the stratigraphic column) of a feature. Only under certain conditions of equal exposure to the modifying agents can the geomorphic age be assumed to be a monotonic function of the geologic age.

Implicit in the concept of geomorphic age is the concept of rejuvenation. On the Earth extreme tectonic activity gives plenty of examples of "pushing back the geomorphic clock'. On the Moon, if all the areas were formed at the same time and progressed through essentially the same history, they should all have the same geomorphic index. In reality the index ranges from approximately 5 to 13 for the mare surfaces and 13 to 20 for the terra surfaces. The simplest way to explain the range in indices is to call for rejuvenative processes, which occasionally wipe out most or all craters in an area.

The most evident process of rejuvenation on the Moon is mare flooding. This process actually creates a new surface, young both geologically and geomorphologically. Impact craters are soon formed and the geomorphic index begins to increase in value. In general, for mare surfaces, the geomorphic index is a monotonic function of the geologic age. Care must be taken in this assumption, however, as a large impact on or near a mare surface will 'geomorphically age' the mare surface prematurely. For example, the area surrounding Copernicus shows a higher geomorphic index than the mare proper, probably as a result of the Copernicus impact. Detailed analysis show, however, that such areas are covered by considerable amounts of ballistic sediments.

Mare flooding is not the only process of rejuvenation. When a large impact occurs, the surrounding area is subjected to the highly erosive action of the ballistic ejecta and seismic activity (the usage of the adjectives large, intermediate and small, when 
applied to impact craters follows closely the definitions presented by Hörz and Ronca, 1971). It was calculated that even if only $10^{-4}$ of the impact energy is converted into seismic energy, a mare-size impact would create moonquakes of approximately magnitude 10 on the Gutenberg-Richter scale, which is considerably larger than any earthquake recorded on Earth. Under the dual attack of the seismic waves and ballistic sediments, crater rims are completely or partially obliterated. From a geomorphological point of view, if no craters or only ghost craters are left, the area will have been rejuvenated. The amount of rejuvenation should decrease progressively as the distance from the impact increases. A clear evidence of this type of rejuvenation is shown by the terrain around Mare Orientale and was discussed in a previous publication (Ronca an Green, 1969).

The absence of craters on slopes surrounding maria (see Gold, p. 55 of these proceedings) gives evidence that rejuvenation may also occur as the result of mass movements produced by the electrostatic action of the solar wind.

The purpose of this paper is to review the assumptions and the methods used to obtain the geomorphic index, to study the relationships between the index and relative time in terms of lunar stratigraphy and to present some conclusions about the length of the interval of time that was necessary to fill up the mare basins with the mare material. It will be shown that this time interval occupies a considerable portion of the lunar geological history. It will also be shown that erosive processes on the highlands are laterally related over large distances, implying a more complicated picture than that offered by erosion by impacts only. Mass movement produced by electrostatic action as described by Gold may be the explanation.

\section{The Geomorphic Index}

The following is a review of material published in Ronca and Green (1968, 1969 and 1970).

The University of Arizona catalog (Arthur et al., 1963, 1964, 1965, 1966) classifies lunar craters on a scale of 1 to 5 on the basis of their condition. Very sharp freshlooking craters are classified as 1 , craters with blurred rims as 2 , craters with extensively broken rims as 3 . Craters usually described as ruins are classified as 4 , and ghost and fragmentary craters as 5 .

No age relationship is intended in the definition of these classes. Intuitively, however, it appears possible that the classes may represent an age sequence. It is possible to perform a test to check the hypothesis that the classes do indeed represent an age sequence. If only those craters larger than a few kilometers are considered (for this size crater saturation is not reached), then, the older a lunar surface is, the more highly cratered it will be. If the classes are a time sequence, then class- 5 craters should be common in highly cratered areas, while class- 1 craters should be common in areas of low crater densities. This is not actually the case. If we plot the percentage of class- 5 craters versus the number of craters per unit area for the craters of the lunar near-side (excluding the limbs) larger than $3.5 \mathrm{~km}$ in diam, we can see that the percen- 
tage of craters which are of class-5 increases to a maximum very quickly for areas of low-intermediate crater densities and finally decreases for areas of high crater densities. Contrary to the hypothesis, areas of high crater density are relatively low in craters of class-5.

We can make a similar test excluding craters of class 4 and 5 . If only craters of class 1, 2, and 3 are considered, then the results fit the hypothesis. The percentage of craters which are of class- 3 is low in areas of low crater density and increases monotonically with the crater density. These relationships can be interpreted to indicate that classes 1, 2, and 3 are a time sequence, while classes 4 and 5 are not.

The next step is to check this interpretation by observing a large number of individual craters. For brevity's sake, only the conclusions will be presented here. They are as follows: All the erosional processes operating on lunar craters can be grouped in two categories. The first category produces a degradation by erosion through time from class 1 to class 3 , and in some cases, class 4 . This can be called the continuous degradation sequence. The second category of erosional agents is responsible for the conditions of craters of class 5 and of some of class 4 . This is not a continuous process, as it can happen to craters belonging to any class. This category will be called the discontinuous degradation. It can also cause rejuvenation, that is, the complete disappearance of craters.

The erosional agents which cause the continuous degradation sequence operate more or less continuously through time (not necessarily at the same rate). Micrometeoritic impact, Gold's electrostatic erosion, and space weathering are likely to be the dominant agents, accompanied by other processes, such as terrace collapse, isostatic recovery and perhaps large-scale tectonics. Specific details of the continuous modification of a crater after its formation have been described by Pike (1967) and Ross (1968).

The erosional agents which cause the discontinuous process are primarily two. Flooding by mare material leaving only a rim or part of a rim above the surface is one. The other is ballistic sedimentation and destruction by seismic waves created by large impacts. If flooding by mare material is so deep as to completely cover the craters of an area or the ballistic sedimentation and seismic waves are so intense as to completely obliterate the craters of an area, the area has been rejuvenated, as previously discussed.

It is evident that if we are interested in a time-related parameter, we must concentrate on the continuous degradation sequence. Craters of class 4 and 5 are, in the great majority, relics of a previous chapter of the geomorphic history of that particular area.

We are now ready to define the geomorphic index. It can be shown that if we plot the logarithm of the percentage of craters of class 1 versus the logarithm of the number of craters per unit area for the craters of the near side larger than $3.5 \mathrm{~km}$ in diam, the data distribute themselves along a line of slope -1 . The following model fits this observation. Let us start with a newly formed surface. For a very short time, it will be without any large crater. Soon impacts will begin to create more and more craters. At first, all craters will be of class 1 , but soon the earliest craters will become class 2 . If 
no large crater and no mare flooding of significance occur to produce any discontinuous degradation, each crater will proceed from class 1 to class 2 and finally to class 3 (a few craters will reach class 4). A newly formed crater will remain in class 1 during the length of time, $t$, necessary for the crater density of the area to increase by a number, $K$, of craters per unit area [note that if the impact flux varies through the lunar geological time (Hartmann 1965, 1966), this length of time, $t$, will not be the same through geologic time]. It can be easily proven that if this model is correct, then the data must distribute themselves on a logarithmic plot on a line of slope -1 , for

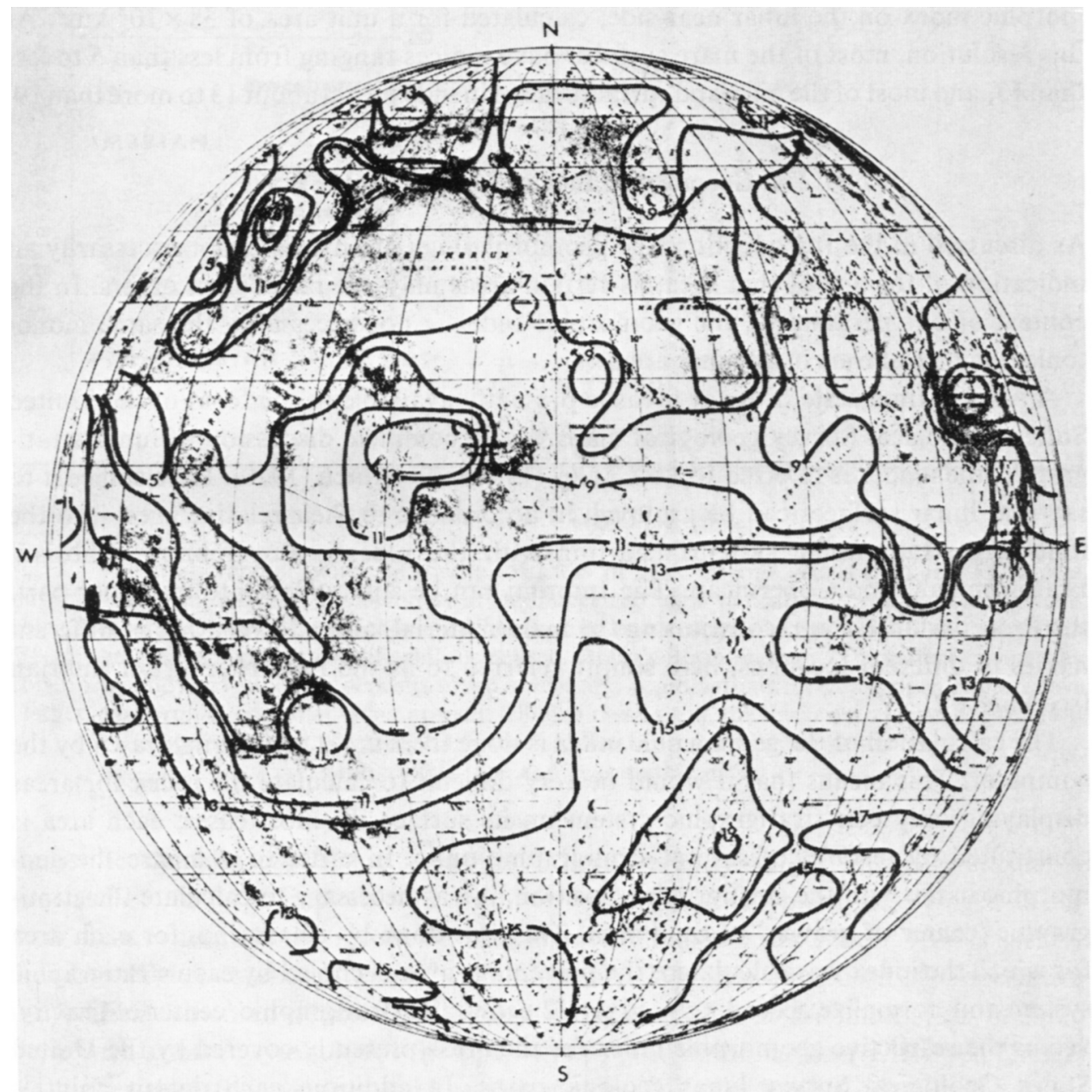

Fig. 1. Countour map of the geomorphic index, calculated for unit areas of $58 \times 10^{3} \mathrm{~km}^{2}$. Apollo 11 landed on $23.49^{\circ} \mathrm{E}, 0.67^{\circ} \mathrm{N}$, on an area of geomorphic index equal to 10.3. Apollo 12 landed on $23.34^{\circ} \mathrm{W}, 2.45^{\circ} \mathrm{S}$, on an area of index equal to 8.4. Luna 16 landed on $56.30^{\circ} \mathrm{E}, 0.68^{\circ} \mathrm{S}$, on an area of geomorphic index equal to 14.3. Although the indices of the landing sites were obtained for areas of $6.4 \times 10^{3} \mathrm{~km}^{2}$, the values fit satisfactorily in the contour map.

Also note the trend in the southern highlands. 
any value of $K$. This is actually the case, as discussed above. The geomorphic index is defined as the position, in arbitrary unit, on the line of slope -1 .

The geomorphic index of a lunar area is more reliable than the crater density or the average crater class because it combines two independently measured parameters crater density and crater class. Although the combination of these two parameters could be obtained more simply by calculating their ratios, this procedure would not take into account the scattering of data. The calculation of the geomorphic index is able to eliminate the scattering not in an arbitrary statistical fashion, but as a direct result of a proposed geological model. Figure 1 shows the contour map of the geomorphic index on the lunar near-side, calculated for a unit area of $58 \times 10^{3} \mathrm{~km}^{2}$. At this resolution, most of the mare surfaces have indices ranging from less than 5 to less than 13, and most of the highland surfaces range in index from about 13 to more than 19.

\section{The Geomorphic Index of Stratigraphic Units}

As discussed in the Introduction, the geomorphology of an area is not necessarily an indication of the age of that area, as certain areas may age faster than others. In the context of our parameters, the geomorphic index is not necessarily the same monotonic function of time in all lunar areas.

A relative time scale of lunar areas is presently available in the form of the United States Geological Survey geological maps. For a complete discussion of lunar stratigraphy, the reader is referred to McCauley (1967) and Mutch (1970). Here suffice it to say that lunar surfaces can be grouped, in accordance to their relative ages, into the following stratigraphic systems (starting with the oldest): Pre-Imbrian, Imbrian, Eratosthenian, and Copernican. The Imbrian can be subdivided into an upper part, the Procellarum Group, constituting the mare material, and a lower part, of different names in different localities, here simply referred to as the Pre-Procellarum Imbrian (see Figure 2).

The calculation of the geomorphic index is done from areas arbitrarily drawn by the computer. This means that it would be very difficult to calculate the index for areas displaying only one stratigraphic system on its surface. In most cases, each area is constituted by terrains of several stratigraphic units. In order to compare the geomorphic index with the stratigraphic position, it was necessary to calculate the stratigraphic 'center of gravity' of each area. This was done by measuring, for each area for which the index was calculated, the percentage area occupied by each stratigraphic system and normalize accordingly. Figure 2 shows the stratigraphic 'center of gravity' versus the respective geomorphic index for the areas presently covered by the United States Geological Survey lunar geologic maps. In addition, each datum point is identified by a different symbol indicating the predominant stratigraphic systems of the area.

Although the plot may not be treated rigorously, it is highly indicative of the relationship between geomorphology and time. Areas rich in Copernican and Eratosthenian terrains are mainly constituted by ballistic sediments produced by recent and 


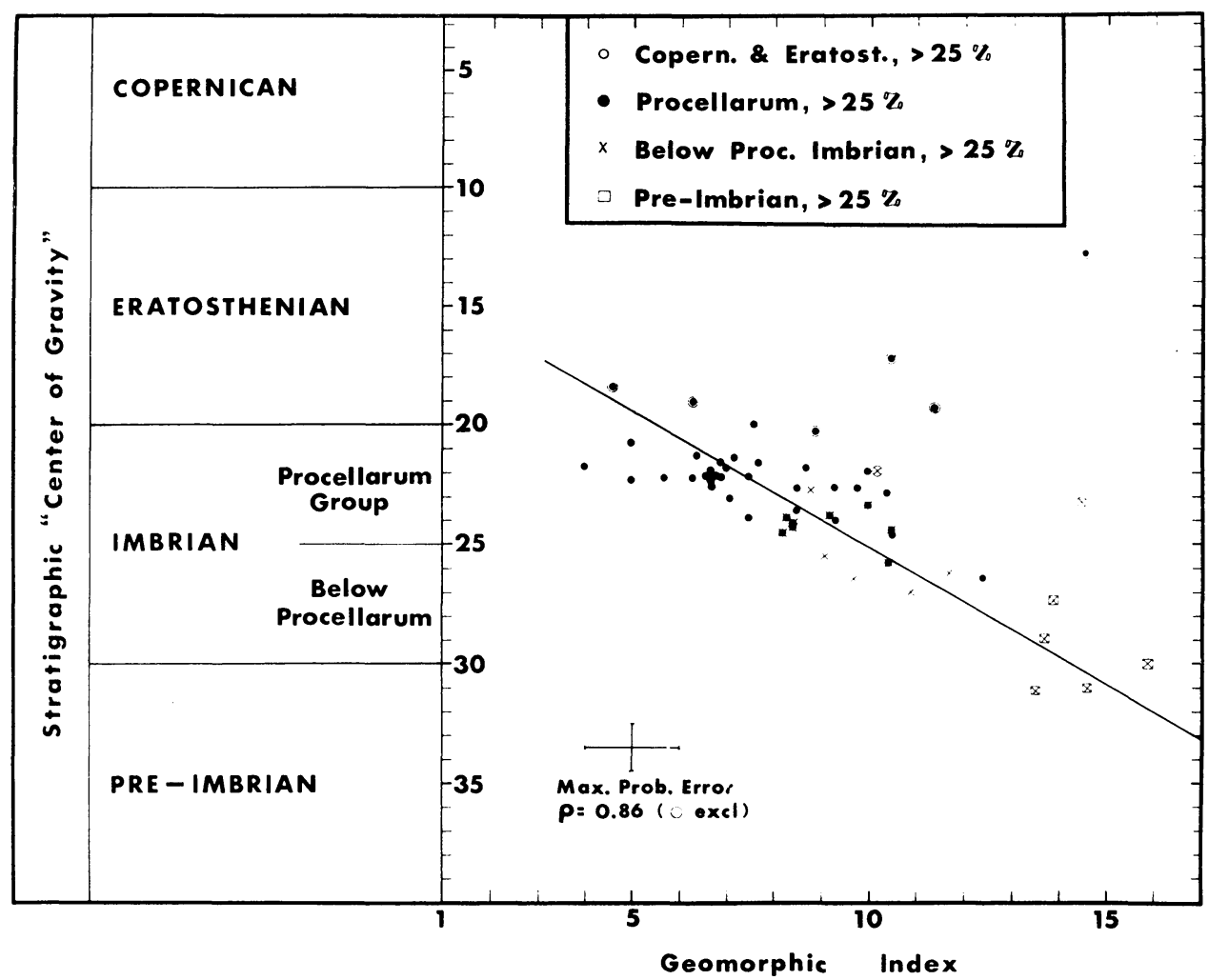

Fig. 2. The stratigraphic 'center of gravity' of areas of $58 \times 10^{3} \mathrm{~km}^{2}$ versus their respective geomorphic index. In addition, the symbols in the upper rectangle indicate the stratigraphic systems which cover more than $25 \%$ of each area. When each of two stratigraphic systems occupy more than $25 \%$ of the area, a combination of symbols is used. Areas with more than $25 \%$ surface covered by Copernican and Eratosthenian deposits (open circles and combinations including open circles) have a larger geomorphic index than other areas. Excluding these areas, the linear correlation coefficient is 0.86 at the $0.1 \%$ probability level, indicating a relationship between stratigraphic time and geomorphic index.

almost recent impacts. These areas have been recently subjected to the effects of intermediate to large impacts and are likely to have geomorphologically aged faster than areas where all the recent or almost recent impacts are small. This is clearly shown in Figure 2, where surfaces with more than $25 \%$ area composed of Copernican and/or Eratosthenian deposits are shown as open circles or a combination of open circle with another symbol. All the open circles (and combinations with open circles) are higher than the average trend, which means that they have a geomorphic index higher than that to be expected by their relatively younger age. This is also indicated in Figure 1 where the contours nest around Copernicus with a higher value than on the maria.

If we exclude the open circles (and combinations with open circles) in Figure 2, the remaining data points correlate well. The linear correlation coefficient between the 
stratigraphic 'center of gravity' (excluding terrains of Copernican and/or Eratosthenian age) and the geomorphic index is 0.86 . If we assume that the data points represent a random sample of all possible data points obtainable from Imbrian and Pre-Imbrian lunar surfaces, then the null hypothesis that there is no correlation between stratigraphic 'center of gravity' and geomorphic index must be rejected at better than the $0.1 \%$ probability level (the chances of making the wrong decision in rejecting the no-correlation hypothesis is less than $0.1 \%$ ).

We can reach the following conclusion: The geomorphic index of areas rich in ballistic sediment is not a monotonic function of time. The intensity and distance of the impact probably plays the most important role. On the other hand, areas rejuvenated by mare flooding (and with an insignificant amount of ballistic sediments) have a geomorphic index which is approximately a monotonic function of time.

\section{The Geomorphic Index of Highland Surfaces}

The distribution of geomorphic indices in the lunar highlands has not been worked out yet and only preliminary observations can be given here. Figure 1 shows how the southern highlands appear to offer an amazing amount of regularity in the contours of the geomorphic index. The farther one goes from the mare shores inland, the higher the geomorphic index becomes. In other words, there appears to be a systematic increase in geomorphic age inland. There is no conceivable process that could explain this trend with an equivalent trend in geological age; processes which could be the cause of this trend, equivalent to ocean floor spreading and continental drift on the Earth, must be completely excluded. The only alternative is that the trend is due to different rates of geomorphological ageing. Areas far away from the shores geomorphologically age faster than areas nearer to the shores.

Presently there is no definitive explanation to this phenomenon. We may add one more observation. Runcorn (p. 377 of these proceedings) shows that the areas of higher geomorphic index in Figure 1 (closed contours 17 and 19 in the southern highlands) correspond closely to areas higher in elevation than the surrounding. Is there a relationship between elevation and rate of geomorphic ageing? One possibility is that the electrostatic erosion mechanism proposed by Gold (see p. 55 of these proceedings) is an important agent of the geomorphological ageing process. Lower areas may be regions of transportation or deposition, while higher areas may be areas of degradation.

It is evident that more research must be done on the geomorphology of highlands. The only comment that can be forwarded is that lunar erosion is not just a localized phenomenon centered on the locus of a large impact, but has lateral trends of regional dimensions.

\section{The Geomorphic Index of Mare Surfaces}

As discussed above, the geomorphic index of mare surfaces uncovered by substantial amounts of ballistic sediments is a monotonic function of time; i.e., the higher the index of a surface, the older the surface is. For the unit area used in preparing the 
contours of Figure 1, $58 \times 10^{3} \mathrm{~km}^{2}$, the index for mare surfaces ranges from less than 5 to less than 13. In order to study the maria in more detail, the unit area was decreased to $6.4 \times 10^{3} \mathrm{~km}^{2}$ and Orbiter photographs and United States Geological Survey geological maps were used to exclude any area that showed a cover of ballistic sediments. A more detailed description was presented in Ronca (1971b).

The range of indices for some of the maria is shown in Figure 3 as a vertical histo-

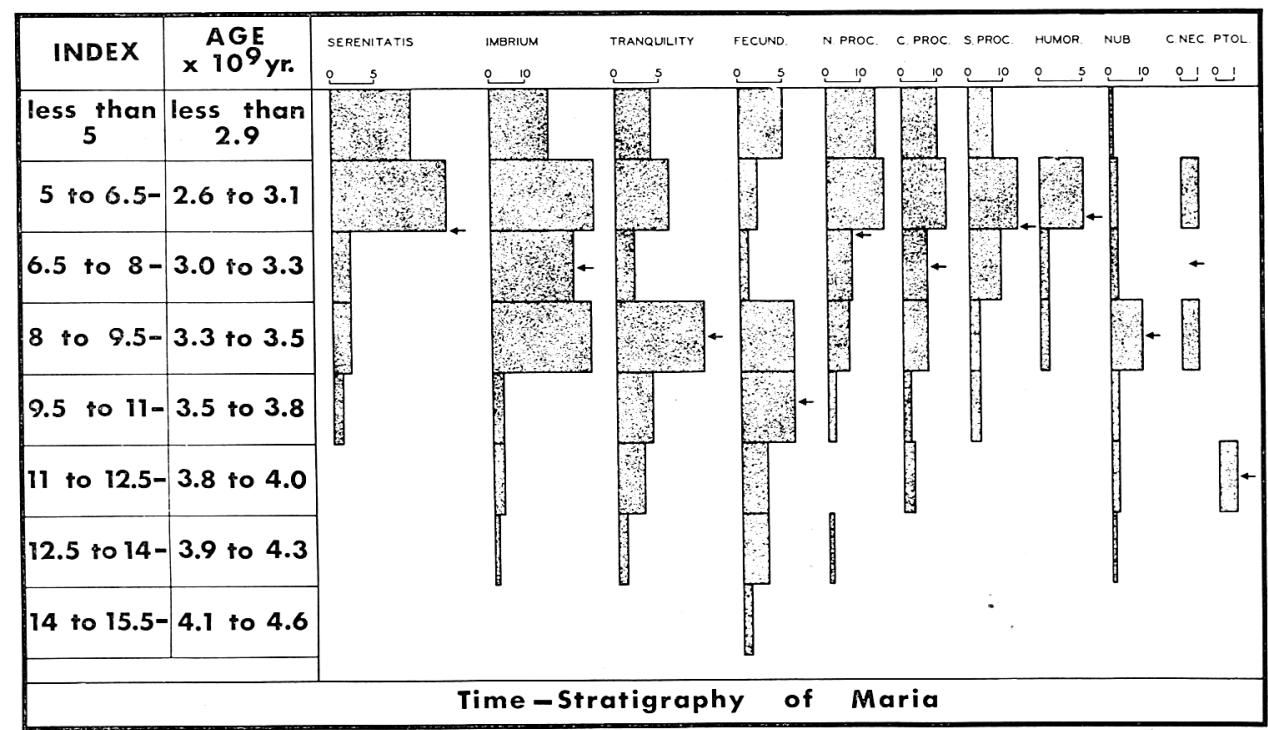

Fig. 3. The distribution of the geomorphic indices for the indicated mare surfaces calculated for unit areas of $6.4 \times 10^{3} \mathrm{~km}^{2}$. The first column to the left shows the values of the geomorphic indices. The second column shows the preliminary range of ages obtained using Figure 4 . The scale under the name of the maria is the number of unit areas displaying the corresponding geomorphic index. Arrows point to the average value for each mare surface. Although the age column is preliminary, it seems likely that the span of time between the oldest and youngest flooding is of the order of $10^{9} \mathrm{yr}$.

gram. Each mare displays a wide range of geomorphic indices, from less than 5 to more than 14. Humorum and Nectaris do not have any geomorphologically young areas (geomorphic indices less than 5).

The following interpretations are offered:

(1) The surfaces of the maria are formed by several floodings having a considerable span of geomorphic indices. This suggests that the filling of the mare basins occupied a considerable length of time.

(2) The geomorphologically oldest surface is found in Fecunditatis. It is however, impossible to state that the flooding activity started sooner in Fecunditatis than in the other maria. The absence or paucity of old surfaces in some maria may simply be due to burial by younger floodings.

(3) No geomorphologically young surfaces are found in Humorum, Nectaris and 
the floor of Ptolemaeus, and Nubium has only a few. A preliminary interpretation is that flooding stopped in some maria sooner than in others.

(4) A tendency toward bimodality in the flooding activity is apparent. The most common surfaces have indices between 5 and 6.5 and between 8 and 9.5. It is impossible to say whether this is significant or is due to statistical fluctuations.

It would be very important to find the functionality of the index of maria versus time. Several researchers have tried to find the relationship between meteoritic flux and time, for a comprehensive summary of all methods the reader is referred to Mutch (1970). But even if the meteoritic flux was exactly known, it would only be of partial help as far as the geomorphic index is concerned. The geomorphic index, as discussed before, is not only a function of cratering but also of the effectiveness of the flux and other agents in the degradation of previously formed craters.

If we assume that the radiometric ages of the samples collected by Apollo 11 and 12 are also the ages of formation of the landing sites, then we can compare the geomorphic indices of the landing sites with the radiometric ages, and have two points in the relationship between these two parameters. The radiometric ages of Apollo 11 and 12 are respectively $3.65 \pm 0.05 \times 10^{9} \mathrm{yr}$ (Albee et al., 1970) and $3.36 \pm 0.1 \times 10^{9} \mathrm{yr}$ (Papanastassiou and Wasserburg, 1970). The geomorphic indices are respectively 10.3 and 8.4 (Ronca, 1971b). Figure 4 shows these points on the age-versus-index

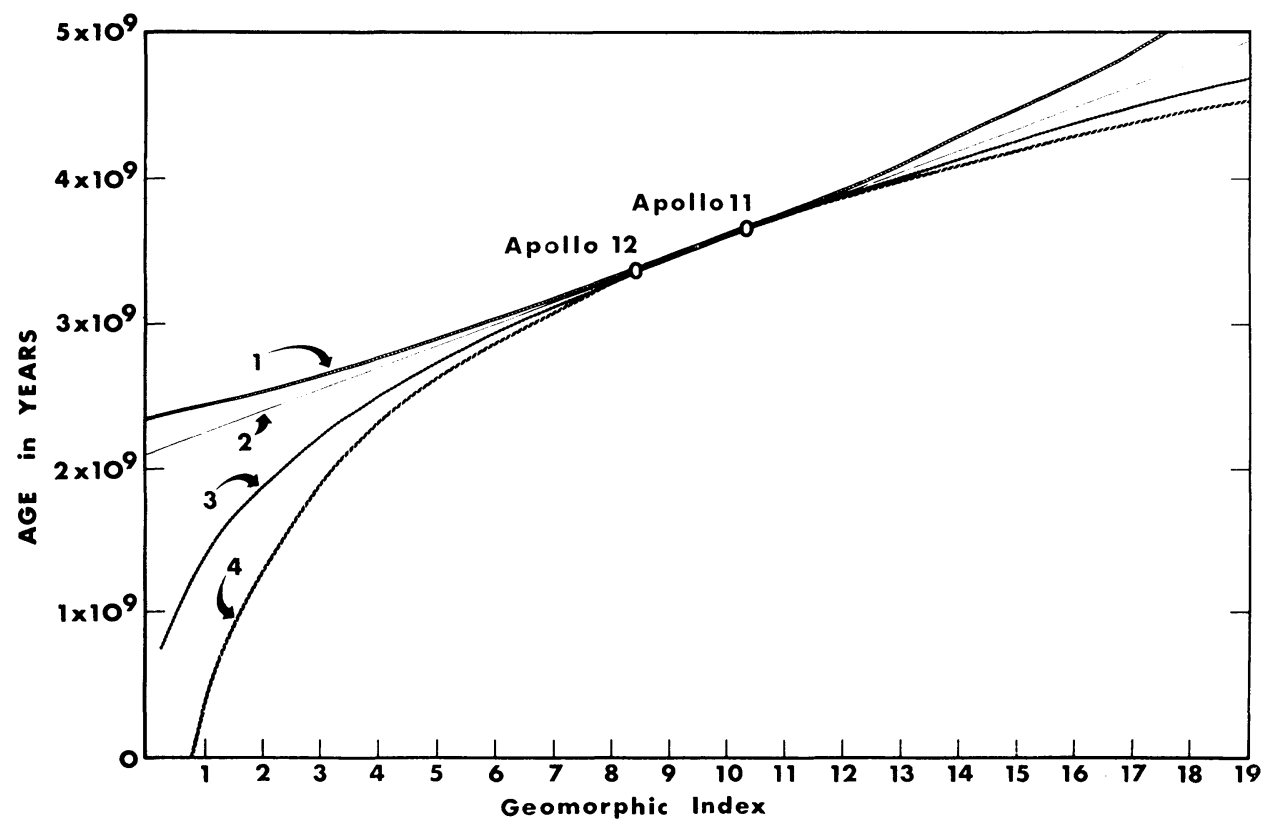

Fig. 4. Geomorphic index versus age, with the values of Apollo 11 and 12 indicated. A linear relationship between the logarithm of age and the index is assumed in curve 1, between age and the index in curve 2 , between the logarithm of age and the logarithm of the index in curve 3 , and between age and the logarithm of the index in curve 4 . If the radiometric age of the basaltic chips collected by Luna 16 is applicable, this age (more than $4 \times 10^{9} \mathrm{yr}$ ) fits the geomorphic index of the landing site (14.3). 
diagram. An infinity of curves can be drawn through the two points, but it is likely that the relationship between index and age is within the limits determined by a linear and logarithmic expression. Four possible curves are drawn: the linear relationship between index and age (curve 2 on Figure 4), between logarithm of the index and age (curve 4), between the index and the logarithm of the age (curve 1) and between the logarithm of the index and the logarithm of the age (curve 3 ). These four curves were also drawn for the limits of the radiometric ages, but are omitted from the figure for clarity. The second column of the vertical histogram of Figure 3 indicates the age limits corresponding to each index interval.

The preliminary conclusions that can be drawn are:

(1) the youngest mare floodings are between $3 \times 10^{9}$ and $0 \mathrm{yr}$ old,

(2) the oldest mare floodings are between $4 \times 10^{9}$ and $4.5 \times 10^{9} \mathrm{yr}$ old,

(3) the flooding activity of the lunar near side lasted for a period of time of the order of one billion years.

Since the drawing of Figure 4 some data have become available from the samples brought back to Earth by the automatic probe Luna 16. The landing area, in Mare Fecunditatis, appears to have a high geomorphic index, approximately 14.3. The radiometric age of the basaltic chips was given to be more than $4 \times 10^{9} \mathrm{yr}$. It is not clear whether this age can be assigned to the soil or the basalt but if it actually refers to the basalt, then there is good agreement between these data and Figure 4.

\section{Acknowledgements}

This paper was prepared under the joint support of The Universities Space Research Association and the National Aeronautics and Space Administration Manned Spacecraft Center, Contract No. NSR 09-051-001, and constitutes The Lunar Science Institute paper No. 51 .

\section{References}

Albee, A. L., Burnett, D. S., Chodos, A. A., Eugster, O. J., Huncke, J. C., Papanastassiou, D. A., Pososek, F. A., Price Russ II, G., Sanz, H. G., Tera, F., and Wasserburg, G. J.: 1970, Science 167, 463.

Arthur, D. W. G., Agnieray, A P., Horvath, R. A., Wood, C. A., and Chapman, C. R.: 1963, 'The System of Lunar Craters, Quadrant 1', Lunar Planet. Lab., Univ. Arizona, Commun. 2, p. 1.

Arthur, D. W. G., Agnieray, A. P., Pellicori, R. H., Wood, C. A., and Weller, T.: 1965, 'The Systems of Lunar Craters, Quadrant III', Lunar Planet. Lab., Univ. Arizona, Commun. 3, p. 1.

Arthur, D. W. G., Pellicori, R. H., and Wood, C. A.: 1966, 'The System of Lunar Craters, Quadrant IV', Lunar Planet. Lab., Univ. Arizona, Commun. 5, p. 1.

Hartmann, W. K.: 1965, Icarus 4, 207.

Hartmann, W. K.: 1966, Icarus 5, 406.

Hörz, F. and Ronca, L. B.: 1971; Modern Geol. 2, 65.

McCauley, J. F.: 1967, in S. K. Runcorn (Ed.), Mantles of the Earth and Terrestrial Planets, John Wiley \& Sons, Inc., New York, p. 431.

Mutch, J. A.: 1970, Geology of the Moon, Princeton University Press, Princeton, U.S.A., 324 pp.

Papanistassiou, D. A. and Wasserburg, G. J.: 1970, Earth Planetary Sci. Letters 8, 269.

Pike, R. J. Jr.: 1967 J. Geophys. Res. 72, 2099.

Ronca, L. B.: 1971a, The Moon 2, 202.

Ronca, L. B.: 1971b, ‘Ages of Lunar Mare Surfaces. The Geological Society of America Bulletin'. 
Ronca, L. B. and Green, R. R.: 1968, Nature 218, 1147.

Ronca, L. B. and Green, R. R.: 1969, Astrophys. Space Sci. $2,22$.

Ronca, L. B. and Green, R. R.: 1970, Geol. Soc. Am. Bull. 81, 337.

Ross, H. P.: 1968, J. Geophys. Res. 73, 1343. 